\title{
Clival Osteomyelitis Presenting as a Skull Base Mass
}

\author{
Matthew Michalowicz ${ }^{1}$ Murugappan Ramanathan $\mathrm{jr}^{2}$ \\ 1 Department of Otolaryngology, Walter Reed National Military \\ Medical Center, Bethesda, Maryland, United States \\ 2 Department of Otolaryngology, Johns Hopkins Medical Institutions, \\ Baltimore, Maryland, United States \\ Address for correspondence Matthew Michalowicz, MD, Department \\ of Otolaryngology, Walter Reed National Military Medical Center, \\ 8901 Rockville Pike, Bethesda, MD 20889, United States \\ (e-mail: matthew.m.michalowicz.mil@mail.mil).
}

J Neurol Surg Rep 2017;78:e93-e95.
Abstract
Keywords
- clival osteomyelitis
- skull base mass
- skull base osteomyelitis
- central skull base osteomyelitis
Central skull base osteomyelitis is a rare, but potentially life-threatening disease entity often lacking otologic symptoms or external auditory canal pathology. We present a case of a man in his 70s who had developed cranial nerve deficits with radiographic evidence that was consistent with a tumor due to this uncommon entity. The radiologic findings and our experience are discussed to increase awareness in the otolaryngology community.

\section{Case Report}

A man in his 70s recently developed headaches along with jaw pain. He underwent a temporal artery biopsy that returned negative for temporal arteritis. The patient endorsed a $20 \mathrm{lb}$ weight loss over the past few weeks. He also noted worsening ptosis of the left eye, fluctuating leftsided ear fullness unresolved by antibiotics, and difficulty with tongue movement causing problems with both swallowing and speaking. His medical history was significant for diabetes well controlled on sitagliptin/metformin. He denied a history of alcohol or tobacco use. On examination, there was an obvious left-sided ptosis, but the pupils were normal. There was no evidence of middle ear effusion. Cranial nerve examination revealed a left-sided motion deficit of the tongue. Nasal endoscopy demonstrated a leftsided nasopharyngeal crust that appeared to be tethered submucosally with evidence of the mucosal erosion of the superior nasopharynx (-Fig. 1). Magnetic resonance imaging (MRI) was obtained, which showed T1 hypointensity, T2 hyperintensity, and enhancement in the basiocciput with an associated rim-enhancing $\mathrm{T} 2$ hyperintense fluid collection in the preclival region with anterior displacement of the posterior nasopharyngeal wall ( $\mathbf{- F i g s . ~} \mathbf{2}$ and $\mathbf{3}$ ). Also, there was an asymmetric enhancement of the soft tissues at the skull base on the left with encasement of the left internal carotid artery and extension of the enhancement to the anterior aspect of the jugular foramen.

The patient was taken to the operating room for an endoscopic endonasal tissue biopsy which diagnosed clival osteomyelitis. He was treated with a 6-week course of intravenous vancomycin and meropenem. His hypoglossal nerve function improved slightly and overall he is doing well.

\section{Discussion}

Skull base osteomyelitis (SBO) is a rare, but potentially lifethreatening disease entity. First described by Meltzer in 1959 as a complication of otitis externa (OE) in an immunocompromised or elderly, diabetic patient. ${ }^{1}$ Chandler further described it as "malignant otitis externa (MOE)." The infection spreads from the ear canal via the fissures of Santorini to the skull base and through venous channels to other portions of the temporal bone, now acceptably called necrotizing otitis externa. ${ }^{3}$ Patients often present with a headache, otalgia, and cranial neuropathies. ${ }^{4}$ Diabetes is often implicated in the disease process due to defects in received

December 5, 2016 accepted after revision March 11, 2017
DOI https://doi.org/

10.1055/s-0037-1602130. ISSN 2193-6366. (c) 2017 Georg Thieme Verlag KG
Stuttgart · New York

License terms

$\Theta(1) \Theta \circledast$ 


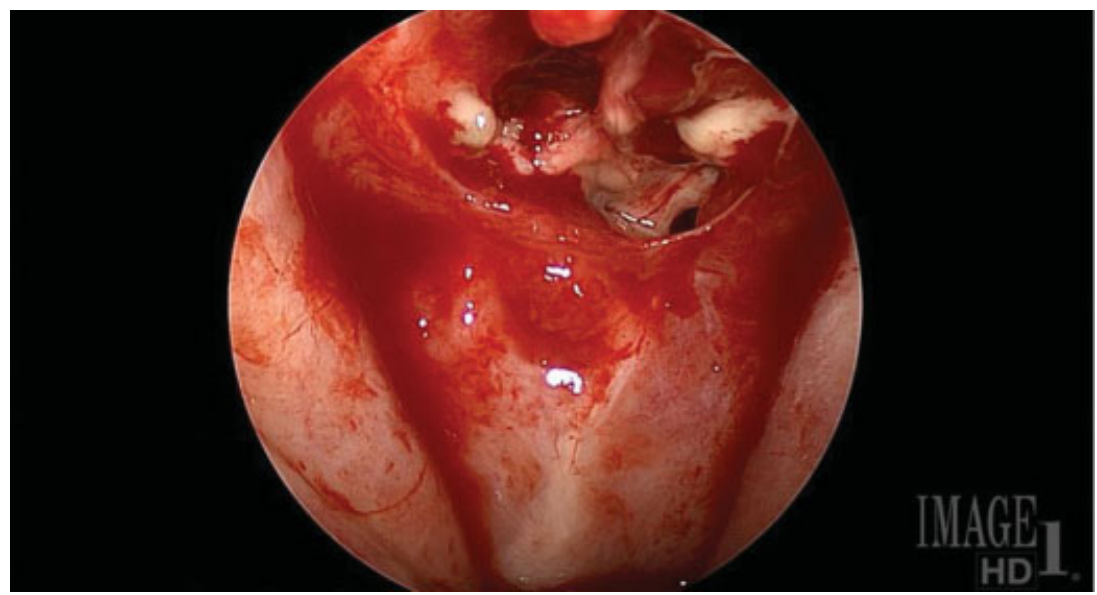

Fig. 1 Preoperative endoscopy of the nasopharynx. Image shows mucopus and granular mass with disruption of mucosal surface.

chemotaxis and phagocytosis. The most common cranial nerve $(\mathrm{CN})$ involved is CN VII. ${ }^{4}$

In central SBO (CSBO), or clival osteomyelitis, there is a lack of otologic symptoms or external auditory canal pathology. The process of "atypical SBO" was first described in 1991 by Sie et al. ${ }^{5}$ In 2003 , Chang et al reported a similar process that did not involve the temporal bone. ${ }^{6}$ Patients typically present with nonspecific headaches or facial pain. Reports vary, but CSBO more often affects the centrally located CN VI, $\mathrm{IX}, \mathrm{X}, \mathrm{XI}$, and XII. Delays in diagnosis are due to vague symptoms and radiographic findings that can mimic central skull base malignancy.

The two most common organisms reported in the literature are Staphylococcus aureus and Pseudomonas aeurginosa. Aspergillosis fumigatus is implicated as the most common fungal pathogen. Recently, Mycobacterium chelonae has been reported due to the rise of acquired immune deficiency

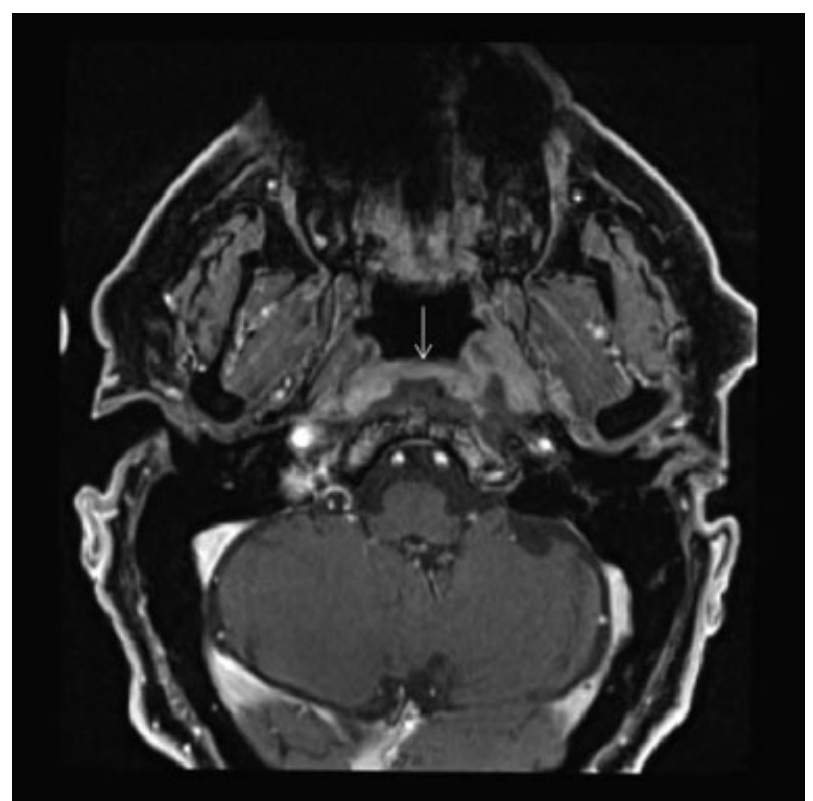

Fig. 2 Axial T1-MRI postgadolinium showing increased uptake of contrast throughout clivus. MRI, magnetic resonance imaging. syndrome and the use of immunosuppressive agents for malignancy. ${ }^{7}$

Initial workup should include a complete blood count with differential, electrolyte panel, and erythrocyte sedimentation rate (ESR). Given the potential radiologic similarities between SBO and malignancy, ESR may be a useful discriminator, as it would not be expected to be elevated in malignancy. ${ }^{8}$

The type of imagining recommended varies from author to author but includes computed tomography (CT), MRI, technetium-99, indium-111, and gallium-67. CT demonstrates diffuse, nonspecific soft tissue swelling, and bony erosion at the central skull base. Typical MRI findings include clival enhancement with hypointensity in the marrow space on T1-weighted images, hyperintensity on T2-weighted images, the presence of dural enhancement, and effacement of the parapharyngeal fat planes, as well as soft tissue masses at the skull base. MRI findings are not specific for an infectious etiology because clival enhancement can also be seen in malignancy. ${ }^{9}$ Technetium-99 is capable of confirming bony involvement and may be the most sensitive for early MOE and osteomyelitis. Technetium studies have largely replaced indium-111 due to shorter acquisition time and better image resolution; however, the findings can be nonspecific and slow to resolve. Gallium-67 has a high specificity

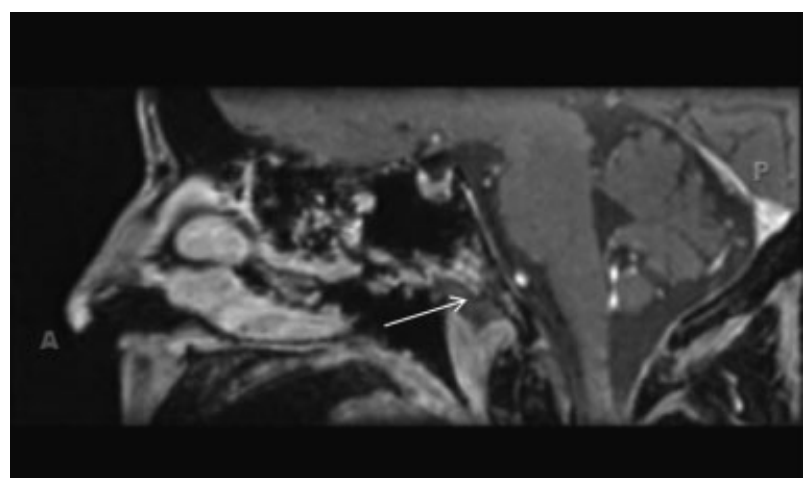

Fig. 3 Sagittal T1-MRI postgadolinium showing increased uptake of contrast throughout clivus. MRI, magnetic resonance imaging. 
for osteomyelitis, and the uptake of gallium resolves more rapidly than technetium. Technetium scans may be useful for diagnosis, but not to determine the duration of treatment. Gallium scans are not useful for diagnosis but can be used to determine the duration of treatment.

Antimicrobial therapy for at least 6 weeks is the mainstay of treatment. As noted in the excellent review by Johnson and Batra, over half of their patients required aggressive treatment with intravenous antibiotics for 6 weeks or longer. Ciprofloxin has historically been the mainstay of treatment although resistance could become a factor in the future. The review also noted that given the possibility of polymicrobial infection, broad-spectrum antimicrobial therapy should be instituted initially until cultures and susceptibility studies become available. ${ }^{7}$ Adjuvant hyperbaric oxygen may also provide some benefit by reversing tissue hypoxia, enhancing phagocytic killing of aerobic microorganisms, and stimulating neomicroangiogenesis, but its availability is limited. ${ }^{8}$

Surgical management should be relegated to biopsy to rule out malignancy. Multiple studies have shown that debridement does not shorten the time of antimicrobial therapy and has no impact on survival, although debridement could be supported if a fungal etiology is suspected. ${ }^{3}$

\section{Disclaimer}

The views expressed in this article are those of the author and do not necessarily reflect the official policy or posi- tion of the Department of the Navy, Army, Air Force, Department of Defense nor the U.S. Government.

\section{References}

1 Meltzer PE, Keleman G. Pyocyaneous osteomyelitis of the temporal bone, mandible, and zygoma. Laryngoscope 1959; 169:1300-1316

2 Chandler JR. Malignant external otitis. Laryngoscope 1968; 78(08):1257-1294

3 Ridder GJ, Breunig C, Kaminsky J, Pfeiffer J. Central skull base osteomyelitis: new insights and implications for diagnosis and treatment. Eur Arch Otorhinolaryngol 2015;272(05):12691276

4 Ganhewa AD, Kuthubutheen J. A diagnostic dilemma of central skull base osteomyelitis mimicking neoplasia in a diabetic patient. BMJ Case Rep 2013;2013:bcr2012007183

5 Sie KC, Glenn MG, Hillel AH, Cummings CW. Osteomyelitis of the skull base, etiology unknown. Otolaryngol Head Neck Surg 1991; 104(02):252-256

6 Chang PC, Fischbein NJ, Holliday RA. Central skull base osteomyelitis in patients without otitis externa: imaging findings. AJNR Am J Neuroradiol 2003;24(07):1310-1316

7 Johnson AK, Batra PS. Central skull base osteomyelitis: an emerging clinical entity. Laryngoscope 2014;124(05):1083-1087

8 Clark MPA, Pretorius PM, Byren I, Milford CA. Central or atypical skull base osteomyelitis: diagnosis and treatment. Skull Base 2009;19(04):247-254

9 Sreepada GS, Kwartler JA. Skull base osteomyelitis secondary to malignant otitis externa. Curr Opin Otolaryngol Head Neck Surg 2003;11(05):316-323 